\title{
Do lean markers relate to exacerbation rate in chronic obstructive pulmonary disease? Preliminary results from AERIS study
}

\author{
M. M. Wojtas ${ }^{1,3,4}$, S. Wootton ${ }^{1,3,4}$, A. Barton ${ }^{1,2,4}$ V. Kim ${ }^{1,2,3}$, K. Ostridge . $^{1,2,3}$, N. Williams ${ }^{1,2,3}$, \\ E. Aris ${ }^{5}$, M. Peeters ${ }^{5}$, J. M. Devaster ${ }^{2}$, S. Bourne ${ }^{1}$ and T. Wilkinson ${ }^{1,2,3}$ \\ ${ }^{1}$ University Hospital Southampton NHS Foundation Trust, Southampton, SO16 6 YD UK, ${ }^{2}$ NIHR Southampton \\ Respiratory Biomedical Research Unit, Southampton, SO16 6 YD UK, ${ }^{3}$ Faculty of Medicine, University of \\ Southampton, Southampton, SO16 6 YD UK, ${ }^{4}$ NIHR Southampton Biomedical Research Centre, Southampton, SO16 \\ $6 Y D$ UK and ${ }^{5}$ GlaxoSmithKline Vaccines, Rixensart, Belgium
}

Previous studies suggested body composition may be related to COPD progression ${ }^{(1)}$ however no definitive studies link lean markers (muscle mass or function) to exacerbation frequency ${ }^{(2)}$. We aimed to investigate this relationship.

An initial cohort of 36 stable COPD subjects (GOLD 2-4) from the AERIS ${ }^{(3)}$ study was analysed. Fat free mass (FFM) was assessed by bioelectrical impedance and function with grip strength (GS), grip endurance (GE) and six minute walk test (6MWT). FFM, impedance at $50 \mathrm{kHz}(\operatorname{Imp50)}$ and GS were adjusted for height and presented as indexes (i). Frequency of exacerbations was prospectively recorded for 12 months and adjusted for length of follow up. A rate of $>0 \cdot 17$ exacerbation/month (i.e. $\geqslant 2$ exacerbations/year) indicated frequent exacerbators. Schols lean depletion cutoffs (FFMi $<16$ male, $<15 \mathrm{female} \mathrm{kg} / \mathrm{m}^{2}$ ) were applied.

$75 \%$ (27) subjects were defined as frequent exacerbators: 53\% moderate, $88 \%$ severe and 100\% very severe COPD subjects. COPD severity and exacerbation rates were similar between genders $(0 \cdot 3$ exacerbations/month).

Women had significantly lower weight, FFM, FFMi, GS and GSi but higher Imp50 and Imp50i than men (all p < 0.05). In women alone, $\mathrm{FEV}_{1} \%$ was significantly related to FFMi $(\mathrm{r}=0 \cdot 52)$ and to 6MWT $(\mathrm{r}=0.61)$. FFMi, GS, GSi and GE were significantly related to 6 MWT $(r=0.49 ; 0.81 ; 0.72 ; 0.49$ respectively).

There were no statistical significant differences for FFM, FFMi, GE, GS, GSi, Imp50, Imp50i, or 6MWT between the frequent and nonfrequent exacerbator groups for either men or women. For men, there could be a trend towards frequent exacerbators for low 6 MWT and high IMP50 values. Using Schols criteria 36\% of subjects ( 2 male, 11 female) were lean deplete, and the exacerbation rates were similar in the 2 subgroups.

Our preliminary results do not provide evidence that FFMi, Imp50, GS or 6MWT are associated with a higher frequency of exacerbation, however Imp50 and 6MWT in men may have predictive value. Lack of statistical significance may be due to the small sample size and high proportion of frequent exacerbators. Further analysis of the full AERIS cohort may yield more information on the predictive value of lean markers on exacerbation frequency and treatment response.

1. Vilaro J, Ramirez-Sarmiento A, Martinez-Llorens JM et al. (2010) Respir Med 104, 1896-1902.

2. Donaldson AV, Maddocks M, Martolini D et al. (2012) Int J Chron Obstruct Pulmon Dis 7, 523-35.

3. Bourne S, Cohet C., Kim V et al. (2014). BMJ Open, 4, e004546. 